\title{
Surface Enrichment in Alloys
}

\author{
TECHNIQUES, THEORY AND PRACTICAL IMPLICATIONS
}

\author{
Robert Bouwman \\ Koninklijke/Shell-Laboratorium, Amsterdam, Netherlands
}

\begin{abstract}
The formation of films of surface active materials on liquid solutions containing them is well known and widely exploited. The segregation or enrichment of one component of an alloy solid solution at its surface is less well known. The techniques which are now available for studying this effect, and the results obtained from their application to gold alloys in particular are reviewed.
\end{abstract}

Gold occupies a distinct place among the elements with regard to chemistry and physics. The main reason is undoubtedly that it is 'inert' to many chemical species in the ambient atmosphere. Articles plated with gold or fabricated from gold alloys are thus thought and, in many cases, prove to be resistant to tarnish and corrosion. Since the onset of degradation of exposed solids occurs at the solid/gas (or liquid) interface, that is at the 'real' surface of the solid, it is not surprising that gold also figures largely in surface chemistry and physics, in particular in catalysis, electrochemistry, optics and electronics.

The purpose of this review is to focus attention on the surface composition of alloys, in particular those containing gold. The constitution of the surface of alloys is a subject of rapidly increasing importance for many branches of industry. The growth to maturity of this part of surface science has been made possible by the almost explosive development of analytical techniques for surface analysis over the last 5 to 10 years. It is therefore of interest to describe the most important practical tools for the analysis of the surface layers of alloys before briefly reviewing the contemporary theories of surface enrichment and finally touching upon the implications of surface enrichment for industrial applications of alloys and for uses of gold alloys in particular.

\section{Techniques for Surface Analysis}

There are nowadays three techniques which are considered to be relatively sound for the determination of solid surface composition. These are:

(1) Electron excited Auger electron spectroscopy (AES)

(2) X-ray photoelectron spectroscopy (XPS), also known as electron spectroscopy for chemical analysis (ESCA)

(3) Low-energy ion scattering (LEIS)
Whichever technique is used, cleaning of the sample and maintaining it in ultra-high vacuum (1.33 $\times 10^{-8}$ to $\left.10^{-9} \mathrm{~Pa}\right)$ are prerequisites for a precise analysis.

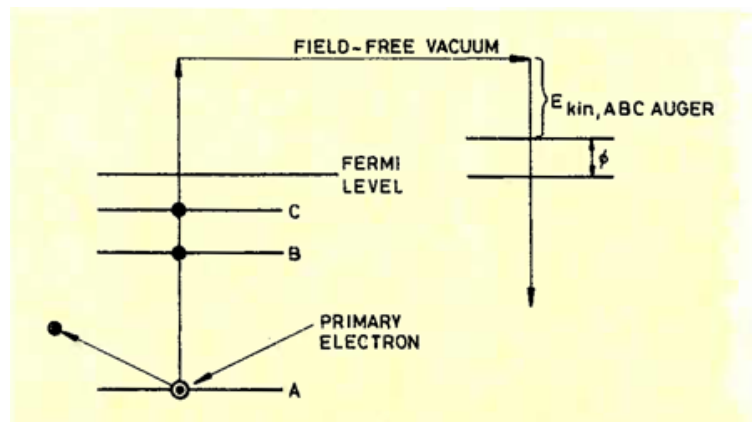

Fig. 1 Schematic representation of the Auger process following the collision of a primary electron with a solid surface

In AES ( 1 to 4), the alloy sample is bombarded with a collimated beam of electrons of typical energy 1 to $10 \mathrm{keV}$ (Figure 1) and the secondary electrons leaving the solid are sorted according to their kinetic energy by means of an energy analyser. One of the processes occurring in the solid upon the electron bombardment is the removal of inner shell electrons. The ions thus produced may decompose by the emission of characteristic photons (fluorescence) or by radiationless transitions. In the latter case, two less tightly bound electrons leave their orbitals through Coulombic repulsion. For instance, one from shell $B$ may fill a primary vacancy created in shell $A$, the other, from shell $\mathrm{C}$ being ejected. The latter is an Auger electron resulting from an $\mathrm{ABC}$ Auger transition. Its energy is calculated from the law of energy conservation as being the difference between the energy of the singly 
ionized initial state $\left(I_{A}\right)$ and that of the doubly ionized final state $\left(\mathrm{I}_{\mathrm{BC}}\right)$ :

$$
E_{A B C}=I_{A}-I_{B C}
$$

If this electron leaves the solid unperturbed, it can be recognised among the mass of other secondary electrons by virtue of its specific kinetic energy:

$$
\mathrm{E}_{\mathrm{kin}, \mathrm{ABC}}=\mathrm{E}_{\mathrm{ABC}}-\phi
$$

where $\phi$ is the work function of the spectrometer entrance. It should be noted that the $E_{k \text { kin }}$ values are finger-print values for the elements present in the solid bombarded.

The majority of Auger electrons do not leave the solid unperturbed. The mean free path of inelastic scattering in solids is so small (Figure 2) that only those Auger electrons which were created in the uppermost 10 atomic layers can be recognised by their $E_{\text {kin }}$ values in the secondary electron spectrum.

In XPS or ESCA $(5,6,7)$ the solid is irradiated by monoenergetic photons, the usual source being aluminium $\mathrm{K} \alpha \mathrm{X}$-rays $(\mathrm{h} v=1486.6 \mathrm{eV})$ or magnesium $\mathrm{K} \alpha \mathrm{X}$-rays $(\mathrm{h} v=1253.6 \mathrm{eV})$. The incident energy is absorbed in the solid by total transfer to core and valence electrons. As is the case with AES, the unperturbed ejected electrons are characterised by their kinetic energy:

$$
\mathrm{E}_{\mathrm{kin}}=\mathrm{h} v-\mathrm{I}_{\mathrm{A}}-\phi
$$

where $I_{A}$ is the ionization energy of shell level $A$ with respect to the Fermi level.

A typical XP spectrum, that of gold obtained with the magnesium $\mathrm{K} \alpha$ radiation, is shown in Figure 3. With expression (3) the ionization energies or fingerprint values for the elements irradiated can be calculated from the observed kinetic energy data.

Like AES, XPS is a surface sensitive technique by virtue of the small mean free path of inelastic scattering of the photoelectrons. Therefore, the intensity of electron lines such as that in Figure 3 provides information on the composition of the surface layers. The high surface sensitivity of the method is demonstrated in Figure 3 by the presence of carbon and oxygen peaks $\left(\mathrm{C}_{1 \mathrm{~s}}\right.$ and $\left.\mathrm{O}_{1 \mathrm{~s}}\right)$ originating from surface contaminants.

XP spectra may also contain Auger lines (see Figure 3) since the shell holes may become de-excited by radiationless transitions. Usually, however, the Auger intensities in XP spectra are rather low.

In LEIS $(8,9)$ the surface of the solid is bombarded with noble gas ions of incident energy $E_{i}$ (typically 1 $\mathrm{keV}$ ). The energy, $E_{f}$, of the ions scattered from the surface under a predetermined angle is analysed. $E_{f}$ is given by the expression:

$$
E_{\mathrm{f}}=\left\{\frac{\cos \theta-\left(\mathrm{r}^{2}-\sin ^{2} \theta\right)^{1 / 2}}{(1+\mathrm{r})}\right\}^{2} \mathrm{E}_{\mathrm{i}}
$$

where $\theta$ is the scattering angle in the laboratory frame and $r=M_{2} / M_{1}$, the ratio of masses of the surfaceexposed atoms $\left(M_{2}\right)$ and the noble gas ion $\left(M_{1}\right)$. Since $\mathrm{E}_{\mathrm{i}}, M_{1}$ and $\theta$ are maintained at fixed values in a given experimental set-up, the energy $E_{\mathrm{f}}$ is determined solely by $M_{2}$. Consequently the energy spectrum of scattered ions is equivalent to a mass spectrum of surface atoms.

Of the three techniques mentioned above AES is used most and LEIS least.

\section{Theory of Surface Enrichment}

The thermodynamic driving force for surface enrichment in alloys is the reduction of the surface free energy of the system. At present, there are two theories which describe surface enrichment of alloys formally, one is the 'bond breaking' theory and the other the 'lattice strain' theory. It is convenient to restrict the discussion of these theories to binary alloys.

The bond-breaking theory considers the difference in heat of sublimation of the elements as the source of the driving force for surface enrichment (10), since the surface energy of metals is proportional to their heat of sublimation. It thus predicts enrichment with those elements which have the lowest heat of sublimation or lowest surface free energy. Experimental indications of its validity were obtained in the period 1965 to $1972(11,12,13)$. Copper-nickel (11), gold-platinum (12) and silver-palladium (13) alloys all exhibited surface enrichment by respectively copper, gold and silver. At that time it was also found (14) as a rule of thumb that such enrichment could be expected to take place at a temperature of roughly $0.3 \mathrm{~T}_{\mathrm{m}}, \mathrm{T}_{\mathrm{n}}$ being the melting point in kelvin of the element responsible for enrichment. More evidence followed for nickel-gold $(15,16)$ and platinum-tin (17) alloys. Confirmation of gold enrichment in gold-platinum at the surface of gold-platinum alloys was published later (18). The theory was further developed in 1974 to $1976(19,20,21)$ and is still considered to be useful.

The lattice strain theory (22) accounts for surface enrichment in substitutional alloys of metals whose atomic radii differ considerably. The strain in the surface lattice is assumed to dominate the effect, with the driving force for surface enrichment being a release of strain energy. Differences in size and compressibility of the atoms in the lattice are thus the parameters of importance. In a practical sense enrichment by the metal with the largest atomic radius is predicted for atomic size differences of at least 10 per cent. Unfortunately there is as yet no unambiguous experimental evidence to support this theory. The gold-copper system is of particular interest in this connection, for whereas the lattice strain theory 


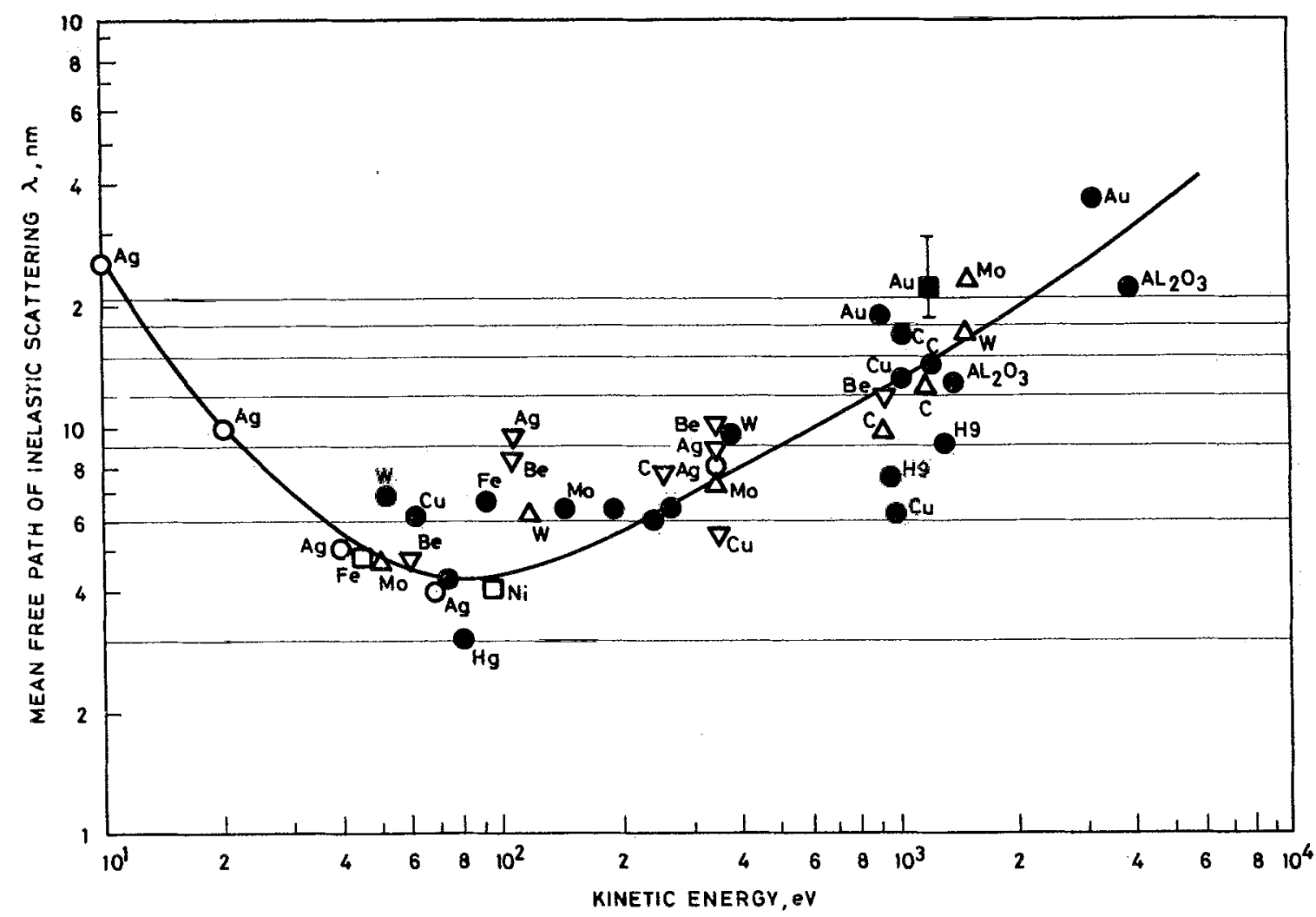

Fig. 2 Mean free path of inelastic scattering $(\lambda)$ of electrons in a solid as a function of their kinetic energy

predicts surface enrichment for gold, which has the larger atomic radius, the bond-breaking theory does so for copper, which has the lower heat of sublimation. Interestingly, results fall into two categories, those which prove gold enrichment $(23,24)$ and those which indicate no enrichment at all $(25,26)$. Enrichment in copper has not been reported so far. Agreement between the experimentalists will probably only be reached by striving for extreme cleanliness of the samples studied.

Studies of the gold-palladium system $(27,28)$ have yielded results compatible with both theories. They have failed to reveal surface enrichment and this is in agreement with the fact that both the heats of sublimation and the atomic radii of palladium and gold are comparable.

It should be noted that all of the above pertains to the surfaces of pure binary alloys equilibrated in vacuum. In 1970, however, it was demonstrated (12) that when the surface of an alloy is exposed not to vacuum, but to another phase, its surface composition may be affected by this phase. Thus, platinum-gold alloys equilibrated in ultra-high vacuum exhibited significant surface enrichment by gold. Subsequent exposure to carbon monoxide, however, resulted in an enrichment of the surfaces of the alloys by

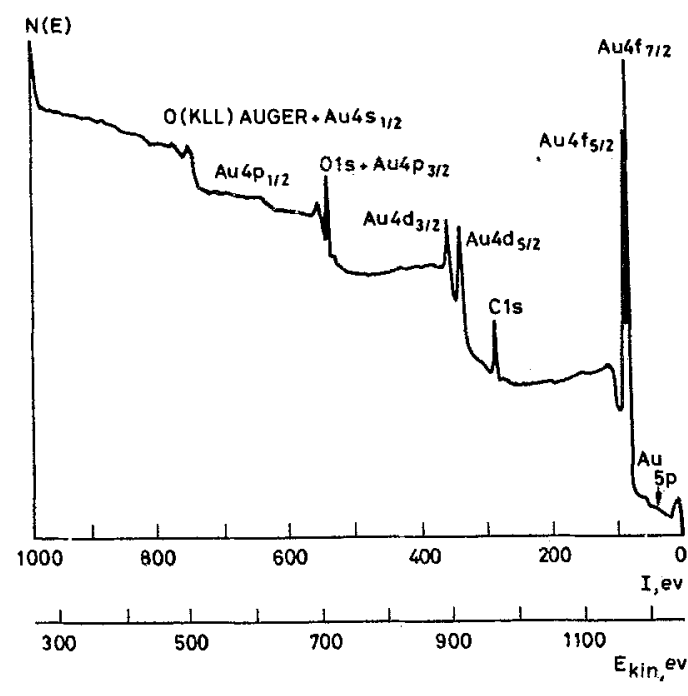

Fig. 3 X-ray photoelectron (XP) spectrum of gold metal obtained with magnesium $\mathrm{K} \alpha$ radiation $(\mathrm{h} v=1253.6$ $\mathrm{eV})$. Surface contamination of this sample is revealed by the presence of $\mathrm{O}_{\mathrm{ls}}$ and $\mathrm{C}_{\mathrm{ls}}$ peaks in the spectrum 


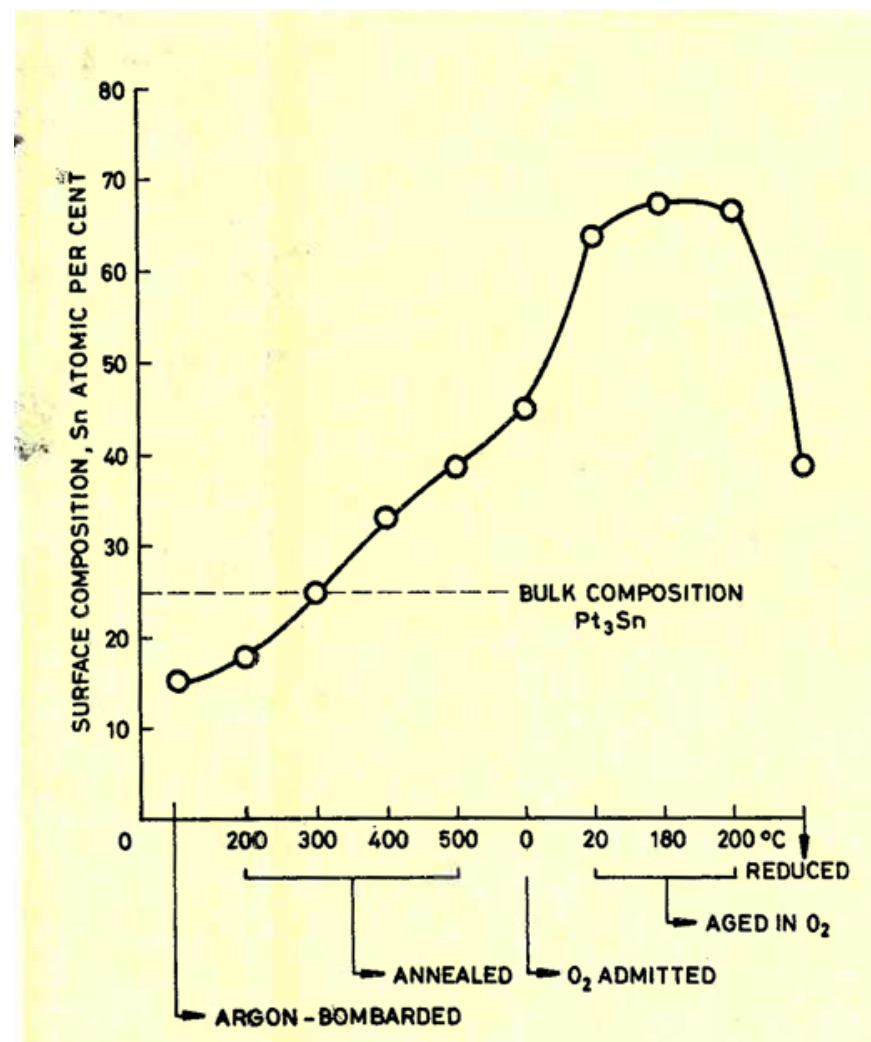

Fig. 4 The extent to which surface segregation may occur in alloy systems is illustrated by this diagram of the changes in surface composition of $\mathrm{Pt}_{3} \mathrm{Sn}$ throughout annealing and chemisorption treatment during which the concentration of tin in the surface layers was observed to vary from 15 to 70 per cent. After (17) platinum. This phenomenon, which was later confirmed in many other systems $(13,15,17,25,28,29$, $30)$, can be qualitatively understood. Carbon monoxide is relatively strongly absorbed on platinum and not on gold at room temperature. Enrichment by platinum at the surface of the alloy gives raise to more Pt-CO bonds and thus decreases the surface free energy. The effect is called 'chemisorption induced surface segregation' and has come to be widely recognised. This type of segregation in a platinum-tin alloy in the presence of oxygen and hydrogen is illustrated in Figure 4.

\section{Practical Implications of Surface Enrichment}

In no branch of surface science has the phenomenon of surface enrichment been studied and used more widely than in heterogeneous catalysis. A comprehensive review of this subject has recently been published (31).

Alloys have played a major role in the understanding and prediction of catalysis by metals since 1950 . However, the following important findings in the mid-sixties boosted the interest in alloy catalysis and surface physics of alloys:

- Many alloy systems of group VIII metals exhibit catalytic and electrochemical synergism for various chemical reactions ( 32 to 36 )
- The selectivity of catalytically active metals can be changed by the addition of other metals, even inactive ones. For example, the selectivity of silver for the oxidation of ethylene to ethylene oxide - an important industrial process - was found to be improved by the addition of a small amount of gold (37).

It is within this context that much research has been conducted on catalysis by alloys. A substantial effort has been invested in the study of alloys of a group VIII metal (nickel, platinum, palladium) with a group IB metal (copper, silver, gold). In this respect, gold plays a key role in catalysis. Itself too inactive to catalyse most chemical reactions satisfactorily, it is very useful as a diluent for the active (group VIII) metal component. An impressive amount of catalytic knowledge is being built up on the basis of the copper-nickel and gold-platinum systems (31). The work done on these systems has produced evidence that alloying of the inactive metal (gold, silver, copper) with the active metal results in a catalyst with a different behaviour towards hydrocarbons, namely a reduced activity for $\mathrm{C}-\mathrm{C}$ bond breaking ('cracking'). Present thinking is that the inactive metal atoms at the surface of the catalyst not only act as spacers which reduce the exposure of the active metal, but also change the electronic structure of the active metal atoms. 


\section{Concluding Remarks}

The factors which lead to differences between the bulk and the surface composition of binary alloys are now reasonably well understood. In many alloy systems the difference in heat of sublimation seems to form the driving force, causing surface enrichment by that metal with the lower heat of sublimation. However, the nature of the gaseous or liquid phases in contact with the surface of the alloy may be such that enrichment by that metal which forms the stronger chemical bond with the adsorbate is given preference.

The study of alloy surfaces, in particular in relation to heterogeneous catalysis, has paved the way to a better understanding of catalysis by metals and possibly to a new generation of selective and stable catalysts tailor-made for specific chemical processes.

\section{References}

I C. C. Chang, in 'Characterization of Solid Surfaces', edited by P. F Kane and G. B. Larrabee, Plenum Press, New York, 1974

2 A. Joshi, L. E. Davis and P. W. Palmberg, in 'Methods of Surface Analysis', edited by A. W. Czanderna, Elsevier, Amsterdam, 1975

3 C. R. Brundle, F. Vac, Sci. Technol., 1974, 11, 212

4 R. Bouwman, Ned. Tijdschr. Vacuumtech., 1973, 11, 37

5 K. Siegbahn et al., 'Atomic, Molecular and Solid State Structure Studied by means of Electron Spectroscopy', ESCA, Almquist and Wiksells, Uppsala, 1967

$6 \mathrm{~S}$. H. Hercules and D. M. Hercules, in 'Characterization of Solid Surfaces', edited by P. F. Kane and G. B. Latrabee, Plenum Press, New York, 1974

7 'Electron Spectroscopy for Surface Analysis', edited by $\mathrm{H}$. Ibach, Springer Verlag, 1977

8 H. H. Brongersma and P. M. Mul, Chem. Phys. Lett., 1972, 14, 380

9 H. H. Brongersma and T. M. Buck, Surf. Sci., 1975, 53, 649
10 J. L. Meijering, Acta Metall., 1966, 14, 251

11 W. M. H. Sachtler and G. J. H. Dorgelo, 9. Catal., 1965, 4, 654 and 4, 665

12 R. Bouwman and W. M. H. Sachtler, 7. Catal, 1972, 19, 127

13 R. Bouwman, G. J. M. Lippits and W. M. H. Sachtler, 7. Catal., 197.2, 25, 350

14 R. Bouwman, and W. M. H. Sachtler, Surf. Sci., 1971, 24, 350

15 F. L. Williams and M. Boudart, f. Catal., 1973, 30, 438

16 J. J. Burton, C. R. Helms and R. S. Polizzotti, F. Chem. Phys., 1976, 65, 1089 and 7. Vac. Sci. Technol., 1976, 13, 204

17 R. Bouwman and P. Biloen, Surf. Sci., 1974, 41, 348

18 J. A. Schwartz, R. S. Polizzotti and J. J. Burton, 7. Vac. Sci. Technol., 1976, 14, 457

19 R. A. van Santen and M. A. M. Boersma, f. Catal., 1974, 34, 13

20 F. L. Williams and D. Nason, Surf. Sci., 1974, 45, 377

21 R. Bouwman, L. H. Toneman, M. A. M. Boersma and R. A. van Santen, Surf. Sci, 1976, 59, 72

22 D. McLean, 'Grain Boundaries in Metals', Clarendon Press, Oxford, 1957

23 J. M. McDavid and S. C. Fain Jr., Surf. Sci., 1975, 52, 61

24 W. Losch and J. Kirschner, in 'Proc. 7th Int. Vac. Congr. and 3rd Int. Conf. Solid Surf.', edited by R. Dobrozemsky, F. Rüdenauer, F, P. Viehbock and A. Breth, Vienna, 1977, 2339

25 R. A. van Santen, L, H. Toneman and R. Bouwman, Surf. Sci, $1975, \mathbf{4 7}, 64$

26 H. C. Potter and J. M. Blakely, J. Vac. Sci. Technol., 1975, 12, 635

27 B. J. Wood and H. Wise, Surf. Sci, 1975, 52, 151

28 G. Maire, L. Hilaire, P. Legare, F. G. Gault and A. O'Cinneide, 7. Catal, 1976, 44, 293

29 P. E. Hoilund Nielsen, Surf. Sci, 1973, 40, 445

30 P. S. Ho, J. E. Lewis and J. K. Howard, f. Vac. Sci. Technol., $1977,44,322$

31 W. M. H. Sachtler and R. A. van Santen, Adv. Catal, 1977, 26, 69

32 D. W. McKee and F. J. Norton, 7. Phys. Chem., 1964, 68, 48

33 G. C. Bond and D. E. Webster, Platinum Met. Rev., 1965, 9, 12; 1965, 9, 14; 1969, 13, 57 and Proc. Chem. Soc., 1964, 398

$34 \mathrm{~J}$. O'M. Bockris and H. Wrobloda, f. Electroanal, Chem. $1964,7,428$

35 O. A. Petrii, Dokl. Akad. Nauk, SSSR, 1965, 160, 871

36 D. V. Sokol'skii, K. K. Dzhardamalieva, A. G. Sarmuzina and T. Tonmarov, Dokl. Akad, Nauk. SSSR, 1967, 176, 1093

37 W. H. Flank and H. C. Beachell, f. Catal., 1967, 8, 316

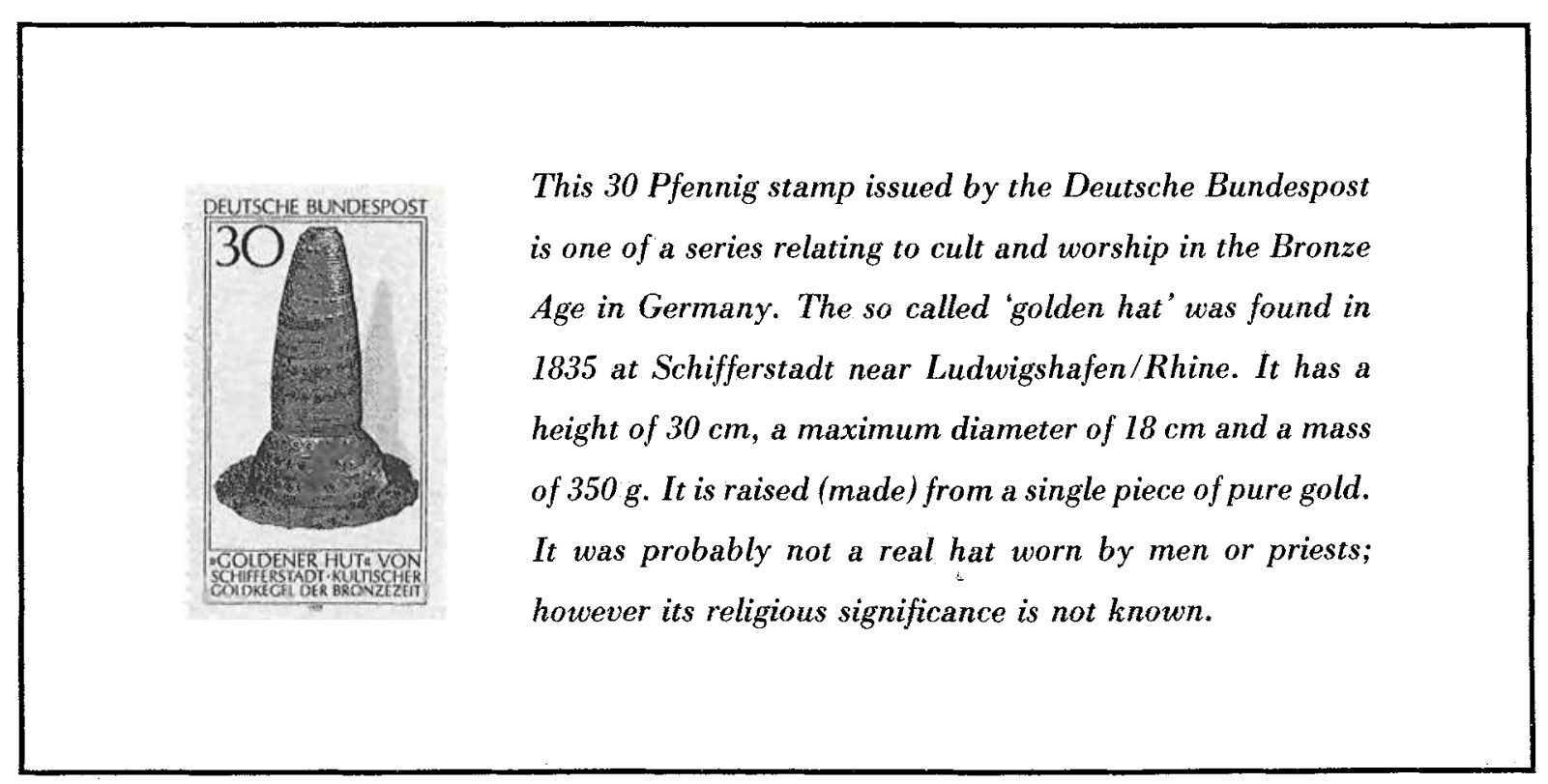

